\title{
¿Existe todavía el derecho a la libertad de cátedra? Dos corrientes polémicas en la academia norteamericana contemporánea*
}

\author{
Raúl Madrid Ramírez**
}

\begin{abstract}
RESUMEN
El objeto de este trabajo es abordar dos de los nuevos límites que parecen presentarse en la actualidad al libre ejercicio del derecho a la libertad de cátedra en la academia norteamericana, que es aquella que genera mayor doctrina al respecto a nivel mundial. Se considerará en primer lugar el concepto de "corrección politica", entendido como una estrategia de dominación del lenguaje, y los efectos que esta originaría en el libre curso de las opiniones científicas. Junto con esta variable, se analiza además las implicancias para la idea de libertad de cátedra del surgimiento de criterios denominados de "justicia académica" que parecen tener la capacidad de debilitar la discrecionalidad de investigación, y sobre todo de difusión de sus resultados. En este último concepto se tiene en cuenta el argumento de que la sociedad se estructura entre opresores y víctimas, de tal manera que la sensibilidad de estas últimas debe ser protegida, incluso en contra del derecho aparentemente acreditado de la libertad de cátedra.
\end{abstract}

Libertad de cátedra - corrección política - universidad

\section{Does the right to academic freedom still exist? Two controversial mainstreams in contemporary north american academy}

\begin{abstract}
The aim of this paper is to consider two of the new possible restrictions to academic freedom in contemporary north american academy, which has developed the biggest worldwide doctrine on the issue. To fulfill this purpose, we will analyze in the first place the notion of "political correctness" as a strategy of language dominance, and its effects on free circulation of scientific opinions. The paper considers also the consequences to academic freedom of the so called "academic justice" emergent criteria, which seems to weaken discretionary research and teaching. It seems
\end{abstract}

* Este trabajo forma parte del Proyecto FONDECYT No 114143 , titulado "El derecho a la libertad de cátedra y la evolución del concepto de universidad. Tres modelos para una misma garantía", en el que el autor se desempeña como investigador principal.

** Licenciado en Derecho, Pontificia Universidad Católica de Chile. Doctor en Derecho, Universidad de Navarra, España. Profesor de Filosofía del Derecho, Pontificia Universidad Católica de Chile. Correo electrónico: rmadrid@uc.cl.

Artículo recibido el 15.3.2017 y aceptado para su publicación en este número el 5.1.2018. 
plausible to discover bebind the concept of "academic justice" the argument that society can be reduced to the binary structure of oppressors and victims, in a way that the victims' sensibility must be publicly protected, even against the apparently well stablished right to academic freedom.

Academic freedom - political correctness - university

\section{El CONCEPTO DE LIBERTAD DE CÁTEDRA}

$\mathrm{L}$ a libertad de cátedra, que comienza su andadura jurídica en el siglo XVIII, es hoy una condición de posibilidad para la investigación, la docencia e incluso la extensión del profesor universitario ${ }^{1}$, y uno de los derechos más apreciados por la sensibilidad democrática en el ámbito intelectual, junto con la tolerancia y la diversidad de puntos de vista. Consiste en la potestad atribuida a los académicos para investigar en cualquier área del conocimiento, y para emitir opiniones científicamente sustentables en su calidad de miembros de una comunidad universitaria, sin que ello traiga sanciones de especie alguna por parte del Estado, de las autoridades universitarias ni de los propios pares. Algunos agregan que esta libertad se extiende también a criticar a la institución para la que se desempeñan ${ }^{2}$. Philip Altbach ${ }^{3}$ la pone en el corazón de la misión universitaria, porque considera a los académicos como miembros de una profesión específica y común -la profesión académica-, con competencias y responsabilidades propias ${ }^{4}$. Esta idea de la profesión común no es nueva, pero adquiere su principal estatus moderno gracias a la Declaration of Principles of Academic Freedom and Academic Tenure de 1915, en la que se sostiene que "si la educación es la piedra angular de la estructura de la sociedad, y si el progreso del conocimiento científico resulta esencial para la civilización, pocas cosas serán más importantes que incrementar la dignidad de la profesión académica, con vistas a atraer hacia sus filas a los hombres de mayor capacidad, de sólido conocimiento y de carácter fuerte e independiente" ${ }^{5}$. Así, la libertad de cátedra "profesional" es aquella relativa al profesor singular, que se enfrenta con poderes vinculados con el Estado o los administradores de las universidades, y no a la autonomía institucional. Protege la emisión de opiniones científicamente fundadas: lo que debe ser juzgado por expertos ${ }^{6}$.

\footnotetext{
${ }^{1}$ Voltaire sostenía que "todo particular que persigue a un hombre, que es su hermano, porque este profesa distinta opinión, es un monstruo”, Diccionario Filosófico, en Obras completas, con prólogo de Victor Hugo, Casa Editorial de M. Senent, Valencia, 1894, vol. IV, p. 794.

2 Andreescu, Liviu, "Individual Academic Freedom and professional Acts", en Educational Theory, vol. $59 \mathrm{~N}^{\circ}$ 5, 2009, p. 559.

${ }^{3}$ Altbach, Philip, “Academic Freedom. International Realities and Challenges”, en Higher Education, vol. 41, No 1/2, Changing Academic Workplace: Comparative Perspectives, enero-marzo de 2001, p. 205.

${ }^{4}$ Atkinson, Richard, "Academic Freedom and the Research University", en Proceedings of the American Philosophical Society, vol. 148, No 2, junio de 2004, p. 195.

51915 Declaration of Principles on Academic Freedom. 2. The Nature of Academic Calling.

${ }^{6}$ Metzger, Walter P., Academic Freedom in the Age of the University, Columbia University Press, Nueva York, 1960, pp. 89-92.
} 
Respecto de esto último es posible dos consideraciones: (a) qué clase de opiniones están cubiertas por dicha garantía, y (b) dónde deben ser emitidas para que esta opere. En relación con el primer punto, para estar amparado por la libertad de cátedra el juicio en cuestión tiene que estar sustentado científicamente. Si se trata de una opinión extra disciplinar; carece de valor científico, y por tanto pertenece al mundo "privado". En 1784, a propósito de una discusión académica acerca de la esencia de la Aufklärung, Kant había propuesto esta distinción, apuntando fundamentalmente a las materias religiosas. El uso de la razón que cabía restringir era el privado, es decir, el que se ejerce bajo una determinada función o puesto civil. Esta distinción abría la posibilidad a los profesores universitarios de mantener, en el plano personal y en sus publicaciones (uso público), una opinión más eventualmente "libre" que en el ejercicio de sus tareas como profesores (uso privado) ${ }^{7}$. El argumento de lo público y lo privado se renueva en la academia norteamericana gracias al debate pertinente al evolucionismo que enfrenta a los académicos durante la segunda mitad del siglo XIX ${ }^{8}$.

En relación con el lugar de emisión de opiniones, se ha entendido tradicionalmente que es la sala de clases, la oficina del profesor, y toda otra instancia en la que se congregan académicos, alumnos o público en general con el objeto de tratar algún tema en el que es factible un discurso académico, sea en la propia universidad, en otra o ante una audiencia externa. La doctrina norteamericana agrega la idea de Freedom of Extramural Expresion, y constituye uno de los problemas teóricos más complejos de los asociados a la idea de la libertad de cátedra9 ${ }^{9}$, resultando muy difícil formular una clarificación funcional de la responsabilidad académica fuera de los recintos institucionales, como lo demuestran innumerables $\operatorname{casos}^{10}$.

La libertad de cátedra ha sido uno de los instrumentos más importantes para la configuración de la universidad contemporánea, y es hoy bastante estándar en todo el mundo occidental, sin perjuicio de los escenarios culturales e ideológicos que puedan serle circunstancialmente adversos. En la actualidad, sin embargo, algunas voces sostienen que, aunque todavía se la defiende en teoría y de manera formal, en la práctica parecería haber perdido vitalidad y relevancia para la comunidad universitaria, de modo que los ideales modernos de libertad de expresión y tolerancia se habrían hecho más opacos, y la universidad se inclinaría a adoptar prácticas que serían incluso menos liberales que las de la sociedad en general ${ }^{11}$. Ya en 1996, Richard Rorty manifestaba su preocupación en torno a que la comprensión de la libertad de cátedra por parte de los claustros había

${ }^{7}$ Kant, Immanuel, Beantwortung der Frage, Was ist der Aufkläurung, Ak VIII, 37.

${ }^{8}$ Metzger, cit., pp. 46 ss.

${ }^{9}$ Finkin, Matthew W. y Post, Robert C., For the Common Good: Principles of American Academic Freedom, Yale University Press, 2009, Edición Kindle, posición 1047.

${ }^{10}$ Scott, Joan W., “The Limits of Academic Freedom”, en Turk, James L (ed.), Academic Freedom in Conflict. The Struggle over Free Speech Rights in the University, James Lorimer \& Company LTd, Publishers, Toronto, 2014, p. 117.

${ }^{11}$ Furedi, Frank, What Happened to the University? A Sociological Exploration of its Infantilisation, Routledge, Londres, 2017, p. vi. 
declinado tanto, que en algunos no era más que una simple tradición popular ${ }^{12}$. La tesis que vamos a sostener aquí es que esta supuesta pérdida de importancia de la libertad de cátedra en la doctrina y la práctica académica norteamericana podría encontrarse unida a dos fenómenos: (a) la supuesta existencia de una estrategia de dominación del lenguaje, conocida como political correctness, y (b) la estandarización de la universidad como una institución eminentemente política. El análisis se centra en el caso norteamericano, por dos razones principales: (a) Estados Unidos es el país que ha desarrollado con mayor profundidad la doctrina de la libertad de cátedra, y sus conclusiones -a causa de la prevalencia de su modelo de universidad en el resto del mundo- se han exportado a todas partes, incluyendo al mundo académico hispanoparlante. Asimismo, (b) es en Norteamérica donde surgiría la idea contemporánea de corrección política, desde donde sería exportada posteriormente a otros espacios universitarios.

\section{CORRECCIÓN POLÍTICA Y LIBERTAD DE CÁTEDRA}

En el ámbito norteamericano, la discusión científica respecto de la corrección política debe ser expurgada con cuidado de lo que podríamos llamar el debate "de trinchera". La expresión political correctness fue utilizada por primera vez en Estados Unidos durante los años cuarenta desde posiciones socialistas, para condenar la actitud de los comunistas norteamericanos, que apoyaban la alianza de Stalin con Hitler. Tiempo después, el uso del concepto continuó representando una forma de ataque interno dentro de las posiciones de izquierda, operando como un modo de distinguir la crítica académica de aquellas que mantenían un supuesto carácter ideológico ${ }^{13}$. A partir de la década de los ochenta, el sintagma comenzó a ser frecuente entre los conservadores, para referirse a las expresiones y contenidos supuestamente contrarios al llamado "sueño americano": derechos individuales, libertad de expresión, igualdad de oportunidades, etc. ${ }^{14}$. La acusación consistía en afirmar que, desde los años sesenta, una nueva generación de académicos se habría hecho con el poder en las universidades; especialmente en las humanidades, pero también en cargos administrativos. Estos profesores tendrían una ideología radical, en que la cultura occidental es representada como violenta ${ }^{15}$, y orientada por prejuicios reaccionarios $^{16}$. De carácter nihilista, la nueva ideología eliminaría cualquier distinción

${ }^{12}$ Rorty, Richard, “Does Academic Freedom Have Philosophical Presuppositions?”, en Menand, Louis (ed.), The Future of Academic Freedom, University of Chicago Press, Chicago, 1996, p. 22.

${ }^{13}$ Feldstein, Richard, Political Correctness. A Response from the Cultural Left, University of Minnesota Press, Minneapolis, 1997, p. 57.

${ }^{14}$ Lea, John, Political Correctness and Higher Education. British and American Perspectives, Routledge, Nueva York, 2009, p. 11.

${ }^{15}$ Cf. Bernstein, Richard, "The Rising Hegemony of the Politically Correct”, artículo en el New York Times del 28 de octubre de 1990: http://www.nytimes.com/1990/10/28/weekinreview/ideas-trends-therising-hegemony-of-the-politically-correct.html? pagewanted =all\&pagewanted $=$ print

${ }^{16}$ AAVV, Debating PC. The Controversy over Political Correctness on College Campuses (Paul Berman, ed.), Nueva York, 1992, p. 105 (edición Kindle). 
posible entre verdad y error ${ }^{17}$. Tal sería la causa de que se hubiera producido al interior de la universidad una atmósfera de sorda represión, pues, invocando la sensibilidad de los terceros supuestamente oprimidos, les exigirían a todos que adhirieran a sus propios códigos de habla o de conducta ${ }^{18}$. De este modo, lo políticamente correcto sería entendido como "sana doctrina", ortodoxia u opinión recta, siendo "correcto" lo afirmado por dichos profesores que se habrían adueñado del lenguaje, como si se tratara de la cúpula del partido que entrega el party-line. Estas acusaciones adoptarían con el tiempo, según sus opositores, una mentalidad mesiánica (salvar a Norteamérica, recuperar la dignidad, etc.).

Como se puede advertir, es necesario tener presente que existen dos dimensiones del debate acerca de la corrección política en el mundo norteamericano: (a) la primera adquiere la forma de un enfrentamiento contingente por la hegemonía cultural, y, como todo intento de ocupar espacios de poder, está sujeto a deformaciones, caricaturas y malentendidos, de los que ambas partes son hasta cierto punto responsables. La segunda (b), busca abordar la cuestión en una perspectiva académica, estimando que el debate sobre la corrección política está vinculado tanto con la naturaleza misma de la democracia contemporánea como con una aproximación epistemológica ${ }^{19}$.

El sintagma "corrección política” entraña una confusión en los términos. El lenguaje posee una clara impronta política, en cuanto es el medio primario en donde se entra en contacto con otros seres racionales, y se administran los espacios privados de poder. Además, existe un uso lingüístico propio de la actividad política, que consiste en la influencia que, mediante un acto de habla, el emisor busca ejercer para la organización de la sociedad, para acceder al poder o mantenerse en él, o bien para ejercer una determinada acción, desde el poder ya alcanzado ${ }^{20}$. En este sentido, toda forma de lenguaje es o puede ser política. Así, la expresión "corrección política” incluye una reducción semántica, pues el sintagma no se refiere a la condición de politicidad de todo lenguaje, sino más bien a las relaciones de individuos dentro de un grupo social que permite que unos determinados miembros del grupo ejerzan el poder sobre los otros; su sentido es equivalente a lo socialmente adecuado, aquello que es admitido por los principios de la sociedad en la que se desenvuelve ${ }^{21}$.

El Oxford Dictionary of New Words define la corrección política como la adecuación a un cuerpo de opiniones liberales o radicales en materias de orden social, caracterizado por la defensa de los puntos de vista aprobados (socialmente), y el rechazo del lenguaje y

\footnotetext{
17 Maranto, Robert, Redding, Richard y Hess, Frederick, (eds.), The Politically Correct University. Problems, Scopes and Reforms, The AEI Press, Washington, 2009, I, 2.

${ }^{18}$ Berman, Paul, Debating PC The Controversy over Political Correctness on College Campuses, Dell Publishing, Nueva York, 1992, p. 121 (edición Kindle).

${ }^{19}$ Lea, p. 19.

${ }^{20}$ Guitart, María Paz, Lenguaje político y lenguaje políticamente correcto en España (con especial atención al discurso parlamentario), Servicio de Publicaciones de la Universidad de Valencia, Valencia, 2005, p. 10.

${ }^{21}$ López, Antonio y De Santiago, Javier, Retórica y comunicación política, Cátedra, Madrid, 2000, p. 36.
} 
del comportamiento considerado discriminatorio u ofensivo ${ }^{22}$. Esta definición contiene dos elementos relevantes: el aspecto ideológico: la corrección política aparece como la adecuación a un cuerpo de opiniones liberales o radicales, y el repudio al lenguaje ofensivo, según el baremo de estos criterios. En la misma línea Hoover y Howard, para quienes se refiere a materias de lenguaje inclusivo, y a la defensa de terminología que no sea sexista, racista o vinculada a categorías etarias; a la insistencia en políticas de discriminación positiva; a evitar el eurocentrismo, así como se refleja en el canon tradicional de literatura; a la aceptación del multiculturalismo como una característica valiosa de la sociedad [norteamericana], y a desmantelar la jerarquía que es controlada por medio de las estructuras de poder del varón blanco ${ }^{23}$. Igualmente, Sykes otorga preeminencia al lenguaje, aportando un elemento más: la corrección política sería parte de una transformación mayor de la sociedad, que se reflejaría en la influencia del lenguaje psicológico sobre el político, lo que produciría una síntesis: la política terapéutica ${ }^{24}$. Wilson, por otra parte, estima que es un mito que hace aparecer algunas ideas triviales e inofensivas como apocalípticas $^{25}$. Browne la define como un sistema de creencias y un canon de pensamiento. Este conjunto de creencias ejercería un control sobre el discurso público, decidiendo qué se puede debatir, y cuáles son los términos de ese debate, así como qué políticas de gobierno resultan aceptables, y cuáles no. Su influencia -argumenta- ha crecido durante las últimas décadas, hasta el punto de que se ha convertido en una de las características más dominantes del discurso público, particularmente en el mundo anglosajón ${ }^{26}$.

De estas definiciones es posible extraer los siguientes elementos del supuesto fenómeno denominado "corrección política": (a) se caracteriza por una centralidad del lenguaje; (b) refleja opiniones liberales que sean al mismo tiempo aprobadas socialmente. Nadie discute este punto; el progresismo sostiene que la idea de corrección política es una exacerbación de la estrategia de izquierdas por parte de los conservadores, pero nadie sostiene que se trata de una adecuación a ideas conservadoras; (c) es un sistema de creencias que busca controlar el discurso; (d) rechaza el lenguaje discriminatorio u ofensivo en contra de las minorías (y por tanto es terapéutico). El lenguaje apropiado debe ser un lenguaje inclusivo: que no sea sexista, racista, o que exprese la inferioridad de cualquier minoría. La corrección política formula un estatuto lingüístico de corrección de la idoneidad de las palabras y expresiones del discurso público, de manera directa (por medio del cambio de palabras concretas), o de manera indirecta (mediante la generación de patrones interpretativos). Este código de habla parece no estar vacío

\footnotetext{
${ }^{22}$ Oxford University Press, 1997, citado por Lea, John, Political Correctness and Higher Education. British and American Perspectives, Routledge, Nueva York, 2009, p. vii.

${ }^{23}$ Hoover, J. D. y Howard, L. A., "The political correctness controversy revisited", American Behavioural Scientist, vol. 38, $\mathrm{N}^{\circ} 7,1995$, p. 963.

${ }^{24}$ Sykes, C. J., A Nation of Victims, St Martin's Press, Nueva York, 1992, p. 164.

${ }^{25}$ Wilson, J. K., The Myth of Political Correctness, Duke University Press, Durham, NC, 1996, p. 2.

${ }^{26}$ Browne, Anthony, The Retreat of Reason. Political correctness and the corruption of public debate in modern Britain, Civitas, The Institute for the Study of Civil Society, Londres, 2006, Edición Kindle, p. 244.
} 
de contenido, sino representar sistemas estructurados de pensamiento cuya nota distintiva es oponerse a las grandes narraciones, ordenadoras y jerárquicas: a la afirmación de verdades universales, a la diferencia sexual biológica como fuente de la condición sexual y a la centralidad o preeminencia de cualquier raza, cultura o religión. Esto se manifiesta en creaciones lingüísticas como significant other (en lugar de la compañía del cónyuge), first people (en lugar de Primera Dama), Visually impaired (en lugar de ciego), etc. El lenguaje políticamente correcto evita las expresiones que juzgan, prefiriendo eufemismos abstractos y polisilábicos. Básicamente se trata de un código de lenguaje, en el que las formas son algunas nuevas (como herstory, femicidio), y otras aparentemente conocidas (como disadvantaged, o gay).

A diferencia de las precedentes formas de ortodoxia, religiosas o políticas, la corrección política no es impuesta desde una autoridad central, como el papado o el gobierno civil. Se trata de una forma de control lingüístico que no deriva de una única fuente, sino de varias fragmentadas. Esta característica denota su talante postmoderno, por la idea de dispersión horizontal de la fuente de origen. Foucault recuerda que el poder es comprendido en la tradición como una regla, ley, prohibición, lo que marca un límite entre lo permitido y prohibido por parte de la autoridad ${ }^{27}$. Marx por el contrario -dice Foucault- considera que no existe un poder, sino varios poderes ${ }^{28}$, varias formas de dominación que operan localmente. Todas estas formas de poder, piensa Foucault, son heterogéneas ${ }^{29}$. Así, la corrección política se configura como una estrategia de poder difusa que consiste en la generación de códigos de habla que indican lo que es "bueno" y lo que es reprobable, corrigiendo de esa forma el discurso público hacia determinadas concepciones ideológicas, lo que contribuye a una politización de todos los espacios sociales y académicos ${ }^{30}$.

El canon de lo "ofensivo" que se utilizaría en la corrección política no es el contemplado en el derecho tradicional, en términos de injurias o calumnias. La idea de la agresión lingüística parece encontrarse vinculada con la pretensión de (a) afirmar algo como hipotético o verdadero desde el punto de vista político, social, jurídico o moral, y (b) que dicha afirmación sea entendida como contraria a alguna minoría. La teoría de la victimización tiene por fundamento una aproximación metodológica desde una perspectiva neomarxista ${ }^{31}$, que consiste en dividir la sociedad en dos principios dialécticos: víctimas y opresores. Encarnar a la "víctima" significa ser herido por un acontecimiento externo, o ser oprimido por un tercero. Esto tiene, desde un punto de vista sociológico,

${ }^{27}$ Foucault, Michel, Microfísica del poder, La Piqueta, Madrid, 1992, p. 149.

${ }^{28}$ Marx, Karl, Das Kapital. Kritik der politischen Ökonomie, 1867, libro II.

${ }^{29}$ Foucault, Michel, Las redes del poder, Prometeo Libros, Buenos Aires, 2014, p. 55. Esta conferencia se dictó en 1976 en la Universidad de Bahía, y fue publicada originalmente en la Revista Barbarie, $\mathrm{N}^{\circ} 4$ y 5 (1981-2).

${ }^{30}$ Friedman, Marilyn, y Narveson, Jan, Political Correctness. For and Against, Rowman \& Littlefields Publishers, Maryland, 1995, pp. 47 ss.

${ }^{31}$ Ellis, Frank, Political Correctness and the Theoretical Struggle. From Lenin and Mao to Marcuse and Foucault, Maxim Institut, Christchirch (Nueva Zelandia), 2004, pp. 29 ss. 
un efecto en cascada, pues como demuestra la experiencia norteamericana, si a algunos grupos se les da protección especial, otros comienzan inmediatamente a solicitarla. Por otra parte, los colectivos que han sido políticamente reconocidos como víctimas comenzarían, según esta tesis, a usar su poder para silenciar aquellos que los critican $^{32}$. Si el poder de policía puede ser usado para silenciar a los críticos, como advirtió Orwell en Animal Farm, las víctimas terminan convirtiéndose en agresores.

En virtud de esta cosmovisión, dicha víctima pasaría a tener un estatus político especial, que carecería de una conexión real o necesaria con un daño o con una opresión. Este modo de victimización, en el que se aplica la "corrección", vendría a ser el resultado de una estrategia política de grupos que buscan obtener un trato preferente, como si fuera un mecanismo de "colectivismo sectario"33. El derecho y la idea de bien común serían instrumentos para asegurar el poder sobre los desfavorecidos. El fenómeno tendría tal envergadura, que algunas de las víctimas no llegarían a tomar conciencia de la injusticia que sufren, y considerarían que está bien ser oprimidos, aceptando su papel en la comunidad; por esta razón, los "mejor informados" tienen la obligación de explicarles que en realidad son explotados. Así, la noción de "víctima" se transforma, y pasa a ser un asunto estructural: siempre serán víctimas, por su condición histórico-cultural de minoría desfavorecida, aunque lo nieguen. El objetivo sería no solo mejorar sus condiciones materiales, sino más bien el reconocimiento permanente del estatus de tal: protección especial y trato preferencial, por los medios coercitivos del Estado. Si esta tesis es real, rompe en el fondo el ideal de igualdad del liberalismo clásico ${ }^{34}$.

Por otra parte, como ya se mencionaba, es posible advertir también un paralelismo entre los postulados del marxismo y de la corrección política ${ }^{35}$, así como es presentada por quienes se quejan de sus efectos. Ambos buscan y comparten el "ideal" de una sociedad "sin clases"; es decir, una sociedad donde los sujetos no solo son iguales ante la ley; sino iguales en sentido material. En este sentido, al igual que la afirmación de que toda la condición humana se reduce a parámetros económicos, la idea políticamente correcta de que toda la sociedad se reduce a la igualdad, tiene un cierto sesgo totalitario. Así como en el marxismo la historia está determinada por la propiedad de los medios de producción, en la corrección política la historia se reduce a la narración de los grupos privilegiados por razón de sexo, raza, etc. Contra las dos clases de desigualdad sería necesario luchar, especialmente desde la universidad. Ambas formas de pensamiento declaran que ciertos grupos son buenos, y otros malvados por definición; sin que interese la acción de sus individuos particulares para efectos de la complejidad de estos juicios.

${ }^{32}$ Green David, We're (Nearly) All Victims Now! How political correctness is undermining our liberal culture, Civitas, Londres, 2006, p. viii.

33 Green, cit., p. 14 ss.

${ }^{34}$ Green, cit., p. 22.

35 Ellis, pp. 7 ss. 
El desarrollo de las ideas de izquierdas en Estados Unidos desde la década de los años sesenta tiene dos fases: una primera, vinculada con los ideales del humanismo liberal, y una segunda, totalmente opuesta, en contra del humanismo liberal, donde se sostiene que la democracia occidental, el racionalismo, la objetividad y la autonomía individual son en realidad instrumentos de subordinación, haciendo aparecer esta subordinación como justicia, según veremos en el apartado siguiente. Esta etapa radical tiene su expresión teórica en la postmodernidad, de acuerdo con esto, toda realidad es funcional a la estructura del lenguaje $\mathrm{e}^{36}$.

La noción contemporánea de corrección política, sin embargo, no proviene ni de los orígenes en el comunismo, ni del desembarco postestructuralista: se trata de una auténtica mutación norteamericana de la filosofía de los años sesenta; una especie de rama que se separa de los ideales parisinos del $68^{37}$. Esta variación original proviene de la misma mentalidad norteamericana, que considera que, en los temas culturales, el mejor modo para clasificar a los individuos es mediante los criterios de raza, etnia y género ${ }^{38}$. Así, de Derrida se toma la noción de que el lenguaje y la literatura son estructuras impersonales que, más que cualquier otro elemento, determina a la sociedad. De Gramsci y Foucault proviene el mecanismo de mirar a la cultura como una arena en la que se lucha por el poder, y la orientación a los grupos marginales. Los psicoanalistas aportan el concepto de la dominación patriarcal y masculina. Todo esto conforma lo que se ha denominado la mélange americaine, o la transmutación norteamericana del ideario de los revolucionarios franceses. La opción por mezclar diversas corrientes está presente en la universidad norteamericana desde el College colonial, mediante los Boards mixtos, y el criterio utilitarista de conseguir donantes de todas las procedencias ${ }^{39}$.

Por su parte, los que no comparten la conclusión de que la corrección política es una estrategia de la izquierda, sostienen que se trata de un mito creado por los conservadores. Gracias a estos, se habría extendido la opinión de que existe una agenda represiva, desarrollada por los académicos en las universidades, que buscarían imponer un ethos radical. Dicha campaña retrataría el intento de hacer ver los derechos de los

${ }^{36}$ Un excelente resumen de la idea de postmodernidad en Welsch, Wolfgang, "Topoi de la postmodernidad", en Fischer, H. R., Retzer, A. y Schweizer, J. (eds.), El final de los grandes proyectos, Gedisa, Barcelona, 1997, pp. 36-56.

${ }^{37}$ Berman, Paul, Debating PC. The Controversy over Political Correctness on College Campuses (Paul Berman, ed.), Nueva York, 1992, p. 268 y ss. (edición Kindle).

38 De hecho, estos son exactamente los criterios que los forjadores de la noción de libertad de cátedra excluyeron conscientemente, apoyando la idea de "universalismo" contra la de "particularismo", es decir, el "universalismo" consistía en la eliminación de los criterios "particulares" (confesión religiosa, raza, nacionalidad, etc.) a la hora de juzgar los méritos del trabajo científico, así como la eliminación de las ventajas no meritorias (conexiones, rango o pertenencia determinados grupos). Este es uno de los elementos que da origen a una de las características de la libertad de cátedra en sentido norteamericano: la idea de la profesión académica como una especie de hermandad de los hombres de ciencia. Metzger, cit., pp. 107-8.

${ }^{39}$ Hofstadter, Richard, Academic Freedom in the Age of College, Transaction Publishers, Nueva Jersey, 1996 (reimpresión), pp. 114 ss. 
menos favorecidos como infracciones a los derechos individuales ${ }^{40}$. La crítica conservadora acusa a sus adversarios de actuar como una especie de policía del pensamiento, lo que conduciría a una especie de "reino de la intolerancia" de proporciones orwellianas, con objeto de silenciar a los que no comparten sus puntos de vista ${ }^{41}$. Sus oponentes responden que los conservadores tendrían paradigmas de comportamiento basados solo en una normalidad unidimensional, en la producción de identidades manufacturadas para el consumo público. La narrativa conservadora caracteriza a estos académicos como autores de códigos represivos y censuradores, porque se dedican a estudiar las estructuras retóricas $^{42}$. Los conservadores -aseguran- usan el miedo; lo que Brennan denomina la "era de la paranoia" ${ }^{43}$. Como dice Girard, para este grupo la corrección política sería una narración en que los académicos [de izquierda] son imputados como un chivo expiatorio, y el lector es inducido a identificarse con el narrador omnisciente, aparentemente incapaz de prejuicios ${ }^{44}$. De este modo, la narrativa de lo políticamente correcto sería, en realidad, mantenida por los ideólogos conservadores ${ }^{45}$, quienes intentan convencer al resto de que su punto de partida es apolítico, neutral. La corrección política que demonizan sería siempre subjetiva, ideológica y dogmática; mientras que, por el otro lado, los "juiciosos” y "objetivos" conservadores defenderían los ideales de una crítica desinteresada, de interés general y con valores trascendentales ${ }^{46}$.

Así pues, dentro del debate norteamericano, los códigos de habla conocidos como estándares de corrección política, o bien son una campaña orquestada desde la derecha política para desacreditar todo lo que desafía los cánones conservadores (incluyendo la discriminación positiva, los derechos de las minorías, el relativismo cognitivo y, en general, las teorías conectadas de algún modo con el neomarxismo), o bien la corrección política es fruto de aquellos situados a la izquierda política, que se ven a sí mismos como los sacerdotes que conducirán a su pueblo hacia la sociedad justa y humana, centrada en la verdadera igualdad entre los hombres, y que está en las antípodas de la retórica política que hoy disfraza las desigualdades.

El núcleo de la discusión acerca del concepto de corrección política es eminentemente universitario. De modo específico, nace y se desarrolla al interior de los centros de educación superior, convirtiéndose en parte del vocabulario moderno y de la mentalidad occidental a raíz del debate que comenzó en el ámbito universitario de Estados Unidos

${ }^{40}$ Feldstein, Richard, Political Correctness. A Response from the Cultural Left, University of Minnesota Press, Minneapolis, 1997, p. X.

${ }^{41}$ Lehman, David. "The Reign of Intolerance”, en Partisan Review, vol. 15, No 4, 1993, p. 598.

${ }^{42}$ Feldstein, cit., p. 2.

${ }^{43}$ Brennan, Teresa, The Transmission of Affect, Cornell University Press, Ithaca, 2004, pp. 185 ss.

${ }^{44}$ Girard, René, The Scapegoat, Johns Hopkins University Press, Baltimore, 1986, p. 40.

45 Lea, cit., p. 14.

${ }^{46}$ Kimball, Roger. Tenured Radicals: How Politics Has Corrupted Our Higher Education, Harper Perennial, Nueva York, 1991, p. 65. 
entre los años ochenta y noventa del siglo pasado ${ }^{47}$. Las obras de Allan Bloom ${ }^{48}$, y de activistas como Dinesh de Souza ${ }^{49}$, o David Horowitz ${ }^{50}$, anunciaban ya la discusión.

Los defensores de la existencia de una estrategia de corrección política en el ámbito académico citan distintos ejemplos de lo que describen. Revisemos algunos. En 1988 se produjo un intenso debate público por el programa de estudios de la Universidad de Stanford, que buscaba sustituir los estudios tradicionales pertinentes a la "civilización occidental" por un área de "multiculturalismo". En noviembre de 2013, un alumno de la Universidad de Marquette que deseaba discutir en clase los argumentos contrarios al matrimonio de personas homosexuales, fue invitado a cambiar de tema por la profesora que guiaba la discusión. Al decirle luego otro alumno que se sentía ofendido por no haber discutido el tema, la responsable comentó que "existen opiniones que no son apropiadas, que son dañosas, como las racistas, sexistas, etc. Si hubiera un homosexual en clase, ¿no sería ofensivo para él si levantaras tu mano y lo pusieras en duda [el matrimonio homosexual]? ${ }^{51}$. En octubre de 2015, el Senado de Alumnos de la Universidad de Kansas votó por prohibir el uso de los pronombres específicos de "género" (he/bim, shel her) de sus propios documentos, por constituir "microagresiones" contra quienes no los $u_{\text {usan }}{ }^{52}$. Quienes no aceptan la influencia de la corrección política en la vida universitaria, argumentan que se trata de exageraciones y casos sacados de contexto ${ }^{53}$.

Las universidades también intentan defenderse de esta aparente red de complejas microofensas que impedirían el trabajo académico, al coartar la libertad de expresión de los académicos. En agosto de 2016, la Universidad de Chicago realizó el movimiento más explícito de los últimos tiempos para oponerse a estas prácticas, enviando una carta a los nuevos alumnos en la que rechazaba algunos de los rasgos distintivos de esta concepción, lo que provocó una tormenta de opiniones a favor y en contra en las redes sociales $^{54}$. La carta comenzaba declarando el compromiso de la Universidad con la li-

${ }^{47}$ Hughes, Geoffrey, Political Correctness. A History of Semantics and Culture, Wiley-Blackwell, West Sussex, 2010.

${ }^{48}$ Bloom, Allan, The Closing of the American Mind, Simon \& Schuster, Nueva York, 1988.

${ }^{49}$ D'Souza, Dinesh, Illiberal Education: The Politics of Race and Sex on Campus, Free Press, Nueva York, 1998.

${ }^{50}$ Horowitz, David y Collier, Peter, Destructive Generation. Second Thoughts About the 60's, Summit Books, Nueva York, 1989.

51 "Marquette professor banned from campus after criticizing classroom gay marriage discussion", https://www.ncronline.org/news/people/marquette-professor-banned-campus-after-criticizing-classroom-gaymarriage-discussion. Consultado el 10 de marzo de 2017.

52 http://www.nationalreview.com/article/429102/most-pc-moments-college-campuses-2015. En esta misma línea, un profesor auxiliar del suscrito narró que, mientras realizaba su Magíster en Harvard durante el 2014, un académico pidió a los alumnos que ellos mismos dijeran qué pronombre debía usarse en su caso. Recientemente (2017), un profesor de la Universidad de Toronto se ha negado a la obligación de hablar sin género: http://nationalpost. $\mathrm{com} /$ news/canada/u-of-t-professor-attacks-political-correctness-in-video-refuses-to-use-genderless-pronouns/ wcm/4c459137-0df6-4a1b-8db7-7a9fb2e9e3f4.

53 Cf. https://www.theguardian.com/us-news/2016/nov/30/political-correctness-how-the -right-invented-phantom-enemy-donald-trump.

${ }^{54}$ Cf. https://newrepublic.com/article/136600/fix-toxic-debate-political-correctness-campus. 
bertad de investigación y de expresión: se anima a los miembros de nuestra comunidad a hablar, escribir, escuchar, discutir y aprender sin miedo a la censura. La civilidad y el respeto mutuo son vitales, y la libertad de expresión no supone el derecho de acosar o amenazar a otros. Esperamos que los miembros de la comunidad participen con rigor en debates, discusiones e incluso en desacuerdos, lo que constituye un desafío para ustedes, a veces puede causar malestar a algunos. "Nuestro compromiso con la libertad de cátedra significa que no apoyamos las así llamadas trigger-warnings. No retiraremos invitaciones a charlistas invitados si sus tópicos resultan controvertidos, como tampoco consentiremos en la creación de intellectual safe spaces" ${ }^{55}$. El concepto de trigger-warning se hizo conocido a partir de las plataformas de blogs, como una forma de proteger a los usuarios de un contenido nocivo que pudiera contribuir a poner en acción enfermedades mentales preexistentes, como fotos de niñas con desórdenes alimenticios, que eventualmente pudieran desencadenar o inspirar a alguien que padece de anorexia. En los últimos años, la noción pasó al ámbito de la docencia, dentro del cual, y en el nombre del bienestar emocional de los propios alumnos, estos exigen crecientemente protección respecto de las palabras y las ideas que les disgustan. A partir del principio de limpiar el campus de palabras e ideas o materias que pueden hacer sentir incómodo u ofender a alguien, por tanto, parece generarse un contexto de limitación a la libertad de cátedra. En este nuevo contexto, el trigger-warning es una especie de alerta que los profesores

${ }^{55}$ Reproducimos aquí parte del Reporte del Comité de Libertad de Expresión de julio de 2014: "Por supuesto, las ideas de los diferentes miembros de la comunidad universitaria usual y naturalmente están en conflicto. Sin embargo, no es propiamente el rol de la Universidad el intentar proteger a los individuos de aquellas ideas y opiniones que les resulten desagradables, antipáticas o, incluso, profundamente ofensivas. Sin perjuicio de que la Universidad valore enormemente la civilidad, y, sin perjuicio también de que todos los miembros de la comunidad universitaria compartan la responsabilidad de mantener un clima de mutuo respeto, las inquietudes sobre la civilidad y el respeto mutuo nunca pueden ser usados como una justificación para clausurar un debate de ideas, por muy ofensivas o desagradables que dichas ideas le puedan resultar a algunos miembros de la comunidad.

La libertad para debatir y discutir sobre los méritos de ideas en contradicción no puede significar, desde luego, que los individuos puedan decir lo que sea que se les ocurra, donde se les ocurra. La Universidad puede restringir expresiones que sean contrarias a la ley, que falsamente difamen a un individuo específico, que constituyan una genuina amenaza o acoso, que injustificadamente invadan la privacidad o los intereses de confidencialidad de manera sustancial o que, de cualquier otra forma, sean incompatibles con el funcionamiento de la propia Universidad. Asimismo, la Universidad razonablemente puede regular el momento, lugar o la manera en que se expresen las ideas para asegurar que no alteren las actividades normales de la Universidad. Pero estas excepciones al principio general de la libertad de expresión son de carácter restringido, resultando de vital importancia que estas excepciones nunca sean invocadas de un modo contrario al compromiso de la Universidad de mantener un debate de ideas completamente libre y abierto. En una palabra, el compromiso fundamental de la Universidad es con el principio que implica que el debate y la deliberación no puedan ser suprimidos porque a algunos, o incluso a la mayoría de los miembros de la comunidad universitaria tales ideas les resulten ofensivas, insensatas, inmorales o erróneas. Le corresponde a los individuos de la comunidad universitaria, y no a la Universidad, como institución, el realizar ese juicio para ellos mismos y el actuar de acuerdo con ese juicio, no suprimiendo el discurso, sino contestando abierta y vigorosamente las ideas a las que se oponen. Sin duda, es parte esencial de la misión de la Universidad el promover la habilidad de los miembros de la comunidad universitaria de formar parte de ese debate y deliberación de una manera responsable y efectiva". 
estarían obligados a hacer si alguna palabra o contenido pudiera causar en algún alumno una violenta respuesta emocional, como por ejemplo si se diera como lectura $E l$ gran Gatsby (F. Scott Fitzgerald), el académico estaría en la obligación de advertir que el libro contiene "misoginia" y "abuso físico", de tal forma que los alumnos que hayan sido previamente victimizados por estas causas (o por otras) puedan elegir no leer la obra, por cuanto podría desencadenar un trauma del pasado ${ }^{56}$. Así también, el 15 de diciembre de 2014, el New Yorker online publicó un artículo acerca de los alumnos de derecho de Harvard que solicitaban a sus profesores no enseñarles el delito de violación, y ni siquiera usar la palabra "violar" para referirse a la idea de "violar la ley", por cuanto ambas cosas causaban "angustia y aflicción" 57 ,

El safe space, por su parte, es una expresión que originalmente indicaba que un profesor, una universidad o un grupo de alumnos no toleraba expresiones o acciones en contra de los movimientos homosexuales o transgénero, pero posteriormente se amplió a todos los individuos "marginalizados", para que pudieran reunirse y compartir esas experiencias de marginalización. Se entiende que esta medida fomenta la inclusión, aumenta la sensibilidad y establece parámetros para determinar cómo y dónde las "conversaciones difíciles" pueden tener lugar. Algunos incluso consideran que los varones blancos deben ser excluidos de estos safe places debido a que "no se puede crear igualdad sin que todos participen de la conversación (y, teóricamente, el varón blanco no sufriría microagresiones, y por tanto no podría conversar sobre el punto $)^{58}$.

Como se puede ver, quienes denuncian la existencia de la corrección política sostienen que hay un enfrentamiento real entre las estrategias de lenguaje y el concepto tradicional de los objetivos de la universidad (la verdad y el conocimiento), para lo que se requiere la libertad de cátedra entendida en este caso como la potestad para comunicar el libre curso de la investigación por medio de la palabra. Los que, por su parte, rechazan la existencia de esta estrategia lingüística, estiman que se trataría de casos muy aislados, que solo serían relevantes si se cede a la teoría de la conspiración que presentan los primeros. La libertad de cátedra, en cualquier caso, sigue siendo una aspiración asociada a los valores modernos de democracia y tolerancia en las universidades norteamericanas, pero su efectividad parece en ocasiones efectivamente reducida en el desarrollo de la vida cotidiana al interior de ellas. La corrección política, mediante el criterio de protección de la sensibilidad de los grupos supuestamente marginalizados, pone el énfasis en la determinación subjetiva de la ofensa, y de esta manera parece restringir de hecho el rango objetivo de lo que se puede discutir dentro de los recintos universitarios.

56 "The Coddling of the American Mind", https://www.theatlantic.com/magazine/archive/2015/09/thecoddling-of-the-americanmind/399356/?utm_source=hpfb Consultado el 11 de marzo de 2017.

57 “The Trouble with Teaching Rape Law”, http://www.newyorker.com/news/news-desk/trouble-teachingrape-law. Consultado el 11 de marzo de 2017.

58 “The new language of protest", http://www.washingtonpost.com/sf/style/2016/05/19/what-collegestudents-mean-when-they-ask-for-safe-spaces-and-trigger-warnings/?utm_term $=.9 f 0364 \mathrm{~b} 77 \mathrm{f} 4 \mathrm{f}$ Consultado el 11 de marzo de 2017. 


\section{LA JUSTICIA POLÍTICA COMO LIMITACIÓN A LA LIBERTAD DE CÁTEDRA}

En febrero de 2014, una alumna senior, editora del Harvard Crimson ${ }^{59}$, publicó en la revista un artículo titulado "The Doctrine of Academic Freedom: Let's give up on academic freedom in favor of justice" 60 . Este texto puede considerarse un manifiesto del nuevo significado que la noción de libertad de cátedra va a adquirir en el apogeo de la universidad norteamericana contemporánea, tanto por la claridad de la exposición como por su alarmante honestidad, que marca una diferencia de énfasis con los modelos de corrección política.

Su punto de partida fue el artículo "I.Q". de Richard J. Herrenstein publicado en The Atlantic Montbly en julio de $1971^{61}$. En él, Herrenstein apoyaba las teorías del psicólogo de Berkeley, Arthur Jensen, el que aseguraba que la inteligencia era casi completamente una facultad hereditaria, y cambiaba de raza en raza. Herrenstein concluía que, por esta razón, los programas destinados a formar una sociedad más igualitaria eran inútiles, porque el modelo dominante estaba basado en diferencias hasta cierto punto heredadas. Al volver a sus clases, fue recibido por un conjunto de alumnos activistas, que incluso solicitaron su despido de la Universidad de Harvard. Herrenstein sostuvo que lo que más le molestaba del asunto era que "algo había ocurrido en Harvard, por cuanto ahora resultaba peligroso para un profesor enseñar cierto tipo de opiniones”. Era justamente lo que buscaban los activistas: conseguir que determinados puntos de vista no fueran bienvenidos. Las autoridades de la Universidad tampoco parecían muy contentas con el incidente, manifestándose preocupadas por la libertad de cátedra y el derecho a expresarse de un miembro del claustro. La editora del Crimson se pregunta entonces: ¿afectaron los activistas la libertad de cátedra de Herrenstein? Y su respuesta es que sí, que efectivamente lo hicieron. Pero luego agrega que esa no es la pregunta más importante que se debe formular, en la medida en que "la obsesión con la doctrina de la libertad de cátedra suele estrellarse contra algo que considero mucho más importante: la justicia académica”. La autora considera que es necesario distinguir dos situaciones: la primera, que, al tenor de las declaraciones de la AAUP, parece correcto en principio que los profesores dispongan de total libertad en la investigación y en la comunicación de sus resultados. Sin embargo - la segunda situación- la obsesión liberal con la libertad de cátedra le resulta inapropiada. Después de todo -argumenta- nadie dispone de "plena libertad" para investigar y publicar, pues los proyectos de investigación que se financian, así como los artículos que se aceptan para publicación, varían según las prioridades políticas. Las palabras que se utilizan para articular una investigación pueden tener consecuencias para su posterior financiación. De esta manera, ninguna cuestión académica es nunca "libre" respecto de las realidades políticas. Si nuestra comunidad

${ }^{59}$ El Harvard Crimson es el periódico interno a un College más antiguo que continúa publicándose. Se fundó en 1873.

${ }^{60}$ Korn, Sandra Y.L., "The doctrine of academic freedom”, en http://www.thecrimson.com/column/ the-red-line/article/2014/2/18/academic-freedom-justice/?, consultado el 3 de septiembre de 2015.

${ }^{61}$ Vol. 228, n. 3, pp. 43-64. 
universitaria, se pregunta, se opone al racismo, al sexismo, al heterosexismo, ¿por qué deberíamos tolerar una investigación que vaya contra nuestros principios, simplemente en nombre de la "libertad de cátedra"? Sandra Korn propone en este punto lo que denomina un "estándar más riguroso: el de la justicia académica”. Este paradigma debiera consistir en lo siguiente: cuando una comunidad académica advierte que una investigación promueve o justifica la opresión, debe asegurarse de que no siga adelante. El poder para hacer cumplir la justicia académica proviene de los alumnos, de las facultades y de los administrativos, todos ellos organizados para conseguir que las universidades se vean como quieren que se vean. Y a continuación cita el caso de un profesor que sostuvo una tesis considerada odiosa, indicando cómo la comunidad de la Universidad de Harvard se organizó para impedirle volver a dar clases. Korn considera este tipo de acciones tanto apropiadas como recomendables, susceptibles de expandirse al sistema universitario en general. Comenta además que estaría muy contenta de organizarse con otras feministas de Harvard para evitar que el profesor Harvey Mansfield siguiera publicando (Mansfield había presentado en 2006 un libro titulado Manliness, que irritó profundamente a los partidarios de las teorías de género, y mereció severas críticas desde algunos medios de comunicación). Korn afirma que "puede que la libertad de cátedra permita estas perspectivas ofensivas, pero la justicia académica no”. La libertad de cátedra aparece así como un obstáculo al argumento político, que consiste en esta noción de justicia ya mencionada. Las personas situadas a la derecha -comenta para terminar-pueden jugar el juego de la "libertad", ya sea económica, de cátedra, etc. Solo quienes se preocupan por la justicia pueden tomar la delantera moral. Aunque sea tentador condenar las restricciones a la libertad de cátedra -concluye-, animaría a los alumnos y a los administrativos de la universidad a utilizar en su lugar un marco de justicia, en la medida en que, si se reduce la obsesión en la doctrina de la libertad de cátedra, se podrá pensar más detenidamente en lo que es justo.

En este comentario está contenida, en nuestra opinión, de manera directa e ingenua la esencia de la concepción postmoderna de la libertad de cátedra. El argumento consiste en atacar al liberalismo, entendido como la idea tradicional de que la libertad de uno termina donde comienza la del otro. Es decir, la regla general del mundo ilustrado y del anglosajón. La razón de ello es porque la autora no concibe nada más conservador que esta modalidad, desde el punto de vista de la organización de la sociedad. Su enemigo, por tanto, es un Estado de Derecho donde se permitan ciertas libertades. El razonamiento que realiza, sin embargo, es un razonamiento clásico -aunque ella misma quedaría muy sorprendida al leer esto-, por cuanto lo que dice es que la libertad debe someterse a un principio anterior, al que llama "justicia". La libertad, en consecuencia, no es el principio rector del orden social, sino más bien algo sustantivo y anterior. Hasta aquí, ninguna diferencia con lo planteado por Aristóteles, en el sentido de que la elección es el resultado de una deliberación previa ${ }^{62}$. La diferencia acontece después, cuando identifica la justicia (es decir, aquello en que consiste la deliberación) con sus intereses

${ }^{62}$ Aristóteles, Ética a Nicómaco, III, 2, 1112a15, y 3, 1113 a11. 
particulares, y no con lo verdadero, en sentido medieval o moderno. La autora, con gran sinceridad, no se siente llamada en cuanto universitaria a una discusión de ideas, sino que da por establecido que la universidad es un escenario de enfrentamiento, y que debe primar en dicha arena la fuerza que sea capaz de imponerse por los hechos. La supresión de la verdad como objeto del pensamiento humano y de la actividad universitaria también es un supuesto en esta argumentación. Korn parece suscribir la posición de Foucault acerca del poder como una relación presente en todo, y considera la universidad como un lugar de enfrentamiento, donde la libertad en sentido moderno es una cortapisa para el triunfo de sus propias ideas. Por esto acepta con toda naturalidad la circunstancia de que las opiniones contrarias deben ser silenciadas: se trata de una cuestión de "justicia”, siendo la justicia lo que es conforme a sus ideas. Aquí se encuentra la justificación del argumento de la superioridad moral que está presente en la mayor parte de los razonamientos de quienes sostienen esta posición, muy vinculado al argumento de las minorías históricamente perjudicadas, al que hacíamos referencia a propósito de la corrección política. La libertad de cátedra es vista ahora como un instrumento de opresión que protege las opiniones dominantes, y por tanto colabora en mantener y profundizar la injusticia con las víctimas y los desprotegidos. Puede ser incluso verdadero que todos tienen derecho a manifestar su opinión (y más todavía si se trata de una clase profesional, experta en sus temas, como son los académicos), pero esto no lo convierte en justo, a los ojos de esta tendencia. Reaparece así la idea de la justicia en sentido material, propia del pensamiento clásico y medieval. Sin embargo, dicha noción se encuentra aquí culturalmente mediada por la influencia postestructuralista, que ha clausurado la posibilidad de apelar a los significados binarios o esencialistas ${ }^{63}$. Por tanto, "justicia” en el discurso de Korn tiene más bien el sentido de coherencia con la lógica dialéctica del enfrentamiento entre víctimas y victimarios, antes que una cuestión estrictamente vinculada con lo que es debido. La justicia es, por tanto, también una estrategia, y en consecuencia, las normas civiles de la sociedad democrática son un obstáculo para los objetivos finales de la acción política implícita. La libertad de cátedra, en este contexto, puede o no ser un verdadero derecho, pero en cuanto problema efectivo para el triunfo del ideario, debe ser removida o al menos no considerada. Esta tesis, tan descarnadamente expuesta por Korn, es mucho más representativa de lo que podría pensarse al leerla, crecientemente también fuera de las fronteras de Estados Unidos. Como dijera Rush Limbaugh (un comentarista político conservador de la cadena de radios Premiere) a propósito del artículo de The Harvard Crimson, "esto no es una casualidad, no es una sátira ni una parodia. Esta mujer es real, y está diciendo en serio que la libertad de expresión debe limitarse si es que amenaza al liberalismo [se refiere a la izquierda]. Quiere decir que no puede ser sometido a crítica" 64 .

${ }^{63}$ Cf. Derrida, Jacques, De la grammatologie, Les Éditions de Minuit, París, 1967. Citamos aquí por la versión española: De la gramatología, (O. del Barco y C. Ceretti, trad.), Siglo XXI argentina editores, 1971, capítulo I.

64 "Harvard writer: abolish free speech", http://www.wnd.com/2014/02/harvard-writer-free -speech-threatens-liberalism/ 
A primera vista, de acuerdo con lo que se ha explicado, la noción de libertad de cátedra contemporánea no parece haber mutado en su definición esencial, sino que se ha visto reducida fuertemente en su extensión, al menos en el modelo de la corrección política. Sin embargo, una mirada más atenta demuestra que tal conclusión no es del todo cierta. La afirmación de Korn y de todas las personas que piensan como ella, en realidad no solo acota la libertad de cátedra, como podía ocurrir mediante la aplicación de estrategias lingüísticas destinadas a erradicar los signos ofensivos a los grupos históricamente desmejorados, sino que produce una variación del paradigma mismo de esta garantía, análogo en importancia al producido en el cambio de modelo que hizo transitar desde la universidad medieval a la moderna. La noción de que existe un criterio de justicia superior al derecho de las ideas a expresarse, supone en cierta forma una recuperación del concepto de verdad en el ámbito moral (es decir no lógico-empírico). Por supuesto, esta "nueva verdad" no tiene nada que ver con el esencialismo metafísico de la universidad escolástica, ni prácticamente tampoco con los parámetros liberales de la universidad moderna.

Parece conveniente, de acuerdo con estas experiencias del ámbito académico norteamericano, reflexionar acerca del estatuto de la libertad académica en el tiempo que viene, y en nuestras propias universidades. La idea moderna de que la libertad de cátedra es el precio que se debe pagar por el bien social de hacer avanzar el conocimiento parece estar ahora en discusión ${ }^{65}$. Sin perjuicio de que la idea de la defensa de las minorías puede ser una cuestión más que atendible, según los casos y las circunstancias, el valor de la libertad de cátedra, como garantía de expresión del pensamiento en contextos tan especializados como las universidades, debe ser defendido seriamente y con rotundidad, tanto contra la monetarización de la universidad como contra su politización ${ }^{66}$. Solo por medio de la libertad de seguir el curso de las investigaciones hacia donde estas nos lleven, y la posibilidad de compartirlas con alumnos que, a su vez, renovarán el pensamiento con su propia reflexión libre, podemos asegurar el futuro de la academia, pero también el futuro de las universidades en un mundo globalizado y multicultural.

\section{BIBLIOGRAFÍA}

Altвach, Philip, "Academic Freedom. International Realities and Challenges", en Higher Education, vol. 41, No 1/2, Changing Academic Workplace: Comparative Perspectives, eneromarzo de 2001, pp. 205-219.

American Association of University Professors, 1915. Declaration of Principles on Academic Freedom.

65 Finkin y Post, p. 44.

66 Tropea, Gregory, "Contingent Faculty and the Problem of Structural Repression”, en Nocella, Anthony J., Best, Steven y McLaren, Peter (eds.), Academic Repression. Reflections from the Academic Industrial Complex, AK Press, Oakland, 2010, p. 479. 
Andreescu, Liviu, "Individual Academic Freedom and professional Acts", en Educational Theory, vol. $59 \mathrm{~N}^{\circ}$ 5, 2009, pp. 559-78.

Aristóteles, Ética a Nicómaco. Utilizamos aquí la versión española Ética a Nicómaco (Julián Marías y María Araujo, trads.), Centro de Estudios Constitucionales, Madrid, 6a edición, 1994.

AtKinson, Richard, "Academic Freedom and the Research University", en Proceedings of the American Philosophical Society, vol. 148, No 2, junio de 2004, pp. 195-204.

Berman, Paul, Debating PC. The Controversy over Political Correctness on College Campuses (Paul Berman, ed.), Nueva York, 1992, p. 268 y ss. (edición Kindle).

Bloom, Allan, The Closing of the American Mind, Simon \& Schuster, Nueva York, 1988.

Brennan, Teresa, The Transmission of Affect, Cornell University Press, Ithaca, 2004.

BRowne, Anthony, The Retreat of Reason. Political correctness and the corruption of public debate in modern Britain, Civitas, The Institute for the Study of Civil Society, Londres, 2006, Edición Kindle.

Derrida, Jacques, De la grammatologie, Les Editions de Minuit, París, 1967. Citamos aquí por la versión española: De la gramatología, (O. del Barco y C. Ceretti, trad.), Siglo XXI Argentina Editores, 1971.

D'Souza, Dinesh, Illiberal Education: The Politics of Race and Sex on Campus, Free Press, Nueva York, 1998.

ElLIs, Frank, Political Correctness and the Theoretical Struggle. From Lenin and Mao to Marcuse and Foucault, Maxim Institut, Christchirch (Nueva Zelandia), 2004.

Feldstein, Richard, Political Correctness. A Response from the Cultural Left, University of Minnesota Press, Minneapolis, 1997.

Finkin, Matthew W. y Post, Robert C., For the Common Good: Principles of American Academic Freedom, Yale University Press, 2009, Edición Kindle.

Foucault, Michel, Las redes del poder, Prometeo Libros, Buenos Aires, 2014.

Foucault, Michel, Microfísica del poder, La Piqueta, Madrid, 1992.

Friedman, Marilyn, y Narveson, Jan, Political Correctness. For and Against, Rowman \& Littlefields Publishers, Maryland, 1995.

Furedi, Frank, What Happened to the University? A Sociological Exploration of its Infantilisation, Routledge, Londres, 2017.

Furner, Mary, Advocacy E Objectivity. A Crisis in the Professionalization of American Social Science. 1865-1905, The University Press of Kentuky, 1975.

Girard, René, The Scapegoat, Johns Hopkins University Press, Baltimore, 1986.

Green, David, We're (Nearly) All Victims Now! How political correctness is undermining our liberal culture, Civitas, Londres, 2006.

Guitart, María Paz, Lenguaje político y lenguaje políticamente correcto en España (con especial atención al discurso parlamentario), Servicio de Publicaciones de la Universidad de Valencia, Valencia, 2005.

Herrenstein, Richard J., "I.Q"., en The Atlantic Monthly, vol. 228, No 3, 1971, pp. 43-64.

Hofstadter, Richard, Academic Freedom in the Age of College, Transaction Publishers, Nueva Jersey, 1996 (reimpresión).

Hoover, J. D. y Howard, L. A., "The political correctness controversy revisited", American Behavioural Scientist, vol. 38, No 7, 1995, pp. 963-975.

Horowitz, David y Collier, Peter, Destructive Generation. Second Thoughts About the 60's, Summit Books, Nueva York, 1989.

Hughes, Geoffrey, Political Correctness. A History of Semantics and Culture, Wiley-Blackwell, West Sussex, 2010.

KANT, Immanuel, Beantwortung der Frage, Was ist der Aufkläurung, 1784. Citamos por la Akademie Ausgabe von Immanuels Kant Gesammelte Werken, vol. X, De Gruyter, Berlín, 1968. 
Kimball, Roger. Tenured Radicals: How Politics Has Corrupted Our Higher Education, Harper Perennial, Nueva York, 1991.

LEA, John, Political Correctness and Higher Education. British and American Perspectives, Routledge, Nueva York, 2009.

Lehman, David. "The Reign of Intolerance", en Partisan Review, vol. 15, No 4, 1993, pp. 598-603. López, Antonio y De Santiago, Javier, Retórica y comunicación política, Cátedra, Madrid, 2000. Maranto, Robert, Redding, Richard y Hess, Frederick (eds.), The Politically Correct University. Problems, Scopes and Reforms, The AEI Press, Washington, 2009.

Marx, Karl, Das Kapital. Kritik der politischen Ökonomie, 1867.

Metzger, Walter P., Academic Freedom in the Age of the University, Columbia University Press, Nueva York, 1960.

Oxford Dictionary of New Words, s.v. "political correctness", Oxford University Press, Oxford, 1997.

Rorty, Richard, "Does Academic Freedom Have Philosophical Presuppositions?", en Menand, Louis (ed.), The Future of Academic Freedom, University of Chicago Press, Chicago, 1996, pp. 21-42.

Scoтt, Joan W., “The Limits of Academic Freedom”, en Turk, James L (ed.), Academic Freedom in Conflict. The Struggle over Free Speech Rights in the University, James Lorimer \& Company LTd, Publishers, Toronto, 2014, pp. 110-126.

Sykes, C. J., A Nation of Victims, St Martin's Press, Nueva York, 1992.

Tropea, Gregory, "Contingent Faculty and the Problem of Structural Repression”, en Nocella, Anthony J., Best, Steven y McLaren, Peter (eds.), Academic Repression. Reflections from the Academic Industrial Complex, AK Press, Oakland, 2010, pp. 479-90.

Voltaire, Diccionario Filosófico, en Obras completas, con prólogo de Víctor Hugo, Casa Editorial de M. Senent, Valencia, 1894, vol. IV.

Welsch, Wolfgang, "Topoi de la postmodernidad”, en Fischer, H. R., Retzer, A. y Schweizer, J. (eds.), El final de los grandes proyectos, Gedisa, Barcelona, 1997, pp. 36-56.

Wilson, J. K., The Myth of Political Correctness, Duke University Press, Durham, NC, 1996.

\section{REFERENCIAS}

Bernstein, Richard, "The Rising Hegemony of the Politically Correct", artículo en el New York Times del 28 de octubre de 1990: http://www.nytimes.com/1990/10/28/weekinreview/ ideas-trends-the-rising-hegemony-of-the-politically-correct.html? pagewanted =all\&pagew anted $=$ print

"MARQUETTE professor banned from campus after criticizing classroom gay marriage discussion", https://www.ncronline.org/news/people/marquette-professor-banned-campus -after-criticizing-classroom-gay-marriage-discussion.

"THE Coddling of the American Mind", https:/www.theatlantic.com/magazine/archive/2015/09/ the-coddling-of-the americanmind/399356/?utm_source $=\mathrm{hpfb}$

"The Trouble with Teaching Rape Law", http://www.newyorker.com/news/news-desk/ trouble-teaching-rape-law

“THE new language of protest”, http://www.washingtonpost.com/sf/style/2016/05/19/what-collegestudents-mean-when-they-ask-for-safe-spaces-and-trigger-warnings/?utm_term $=.9 \mathrm{f} 0364 \mathrm{~b} 77 \mathrm{f} 4 \mathrm{f}$

KoRn, Sandra Y.L., "The doctrine of academic freedom", en http://www.thecrimson.com/column/ the-red-line/article/2014/2/18/academic-freedom-justice/ 
"HARVARD writer: abolish free speech", http://www.wnd.com/2014/02/harvard-writer-free-speech -threatens-liberalism/

“THE thirteen most ridiculously PC Moments on College Campuses” http://www.nationalreview. com/article/429102/most-pc-moments-college-campuses-2015.

http://nationalpost.com/news/canada/u-of-t-professor-attacks-political-correctness-in-video -refuses-to-use-genderless-pronouns/wcm/4c459137-0df6-4a1b-8db7-7a9fb2e9e3f4.

https://www.theguardian.com/us-news/2016/nov/30/political-correctness-how-the-right-inventedphantom-enemy-donald-trump.

https://newrepublic.com/article/136600/fix-toxic-debate-political-correctness-campus. 